\title{
Impacto de un programa de educación ambiental en el manejo de los residuales sólidos peligrosos
}

\section{Impact of an Environmental Education Program on Hazardous Waste Management}

\author{
Daneysis Vera Núñez ${ }^{1 *}$, Edel Castellanos Sánchez ${ }^{1}$, Pedro H. Rodríguez Sanabria ${ }^{1}$ y Mercedes Reyes Navarro ${ }^{1}$ \\ ${ }^{1}$ Hospital Mártires del 9 de Abril, Cuba \\ *daneisyvn@infomed.sld.cu
}

DOI: https://doi.org/10.26871/killkana_salud.v4i2.641

\begin{abstract}
Resumen
Introducción: El riesgo potencial presentado por los residuos sólidos hospitalarios, constituye un problema en términos de salud pública, saneamiento ambiental, enfermedades nosocomiales y epidemiológicos. Es responsabilidad de las instituciones prestadoras de servicios de salud prevenir y contribuir a minimizar este riesgo ambiental. Objetivo: Evaluar el impacto de una educación ambiental en el manejo de los residuales sólidos peligrosos en el Hospital Universitario "Mártires del 9 de Abril"durante el año 2019. Metodología: investigación cuasiexperimental de intervención con antes y después. Se evaluaron servicios de mayor generación de residuales y 269 individuos que estuvieron en los servicios de hospitalización con la totalidad de trabajadores del departamento de Epidemiología. Resultados: el $64.68 \%$ del sexo femenino, de ocupación enfermeros en un $53.15 \%$. De 26 criterios evaluados, antes de aplicado el programa solo 8 recibieron evaluación satisfactoria para un $30.76 \%$, después recibieron evaluación satisfactoria 21 criterios para un $80.76 \%$. Excepto en las etapas del manejo de los residuales solidos peligrosos el resto de los aspectos evaluados no alcanzó más del $49.81 \%$, después alcanzó más del $90.33 \%$ en todos los aspectos evaluados. El impacto del programa resultó ser de $95.16 \%$. Conclusiones: El programa de educación ambiental elevó el nivel de conocimiento de los trabajadores en el manejo de los residuales peligrosos y contribuyó a mejorar los criterios evaluados en las dimensiones, estructura, proceso y resultado en el ambiente hospitalario.
\end{abstract}

Palabras clave: desechos peligrosos, rutas de evacuación, manejo de desechos, plan de rutas de evacuación de los desechos peligrosos.

\begin{abstract}
Introduction: The potential risk presented by hospital solid waste constitutes a problem in terms of public health, environmental sanitation, nosocomial and epidemiological diseases. It is the health services provider institutions' responsibility to prevent and help minimize this ecological risk. Objective: To evaluate the impact of environmental education on hazardous solid waste management at the University Hospital "Mártires del 9 de Abril" during 2019. Methodology: This is a quasiexperimental research with pre- and post-intervention. The highest residual generation services were evaluated, 269 individuals from the hospitalization services participated, with all workers from the Epidemiology unit. Results: Out of 26 assessed criteria, before the programme implementation, only 8 received a satisfactory evaluation of $30.76 \%$; afterwards, 21 of them received a satisfying assessment of $80.76 \%$. Except for the hazardous solid waste management stages, the other aspects evaluated did not reach more than 49.81\%; after implementation, all aspects assessed reached more than 90.33\%. The program impact resulted in being 95.16\%. Conclusions: The environmental education program improved the workers' knowledge level on hazardous waste management, it also contributed to enhancing the criteria evaluated in terms of dimensions, structure, process, and result in the hospital environment.
\end{abstract}

Keywords: hazardous waste, disposal routes, waste management, hazardous waste disposal route plan.

\section{Introducción}

El manejo de los residuos sólidos generados en los servicios médicos públicos y privados (hospitales, centros de salud, policlínicos, postas de salud, consultorios médicos) en los últimos años se ha convertido en un determinante de la calidad de gestión de los mismos que ha sido impulsado sobre todo por el desarrollo de la seguridad y salud en el trabajo, la protección del medio ambiente y la calidad de la atención en los servicios de salud. ${ }^{1}$

Los problemas asociados a los residuos generados por los centros hospitalarios, han sido motivo de preocupación internacional. Dicha motivación ocurre debido al amplio 
espectro de peligrosidad, que ocurre desde la potencial propagación de enfermedades infecciosas, hasta riesgos ambientales derivados de los métodos empleados para su tratamiento y disposición final. Es por ello que la problemática ha trascendido el campo técnico sanitario y ha involucrado aspectos sociales, económicos, políticos y ambientales. El mal manejo de los residuos hospitalarios representa un riesgo para las personas y el medio ambiente por la presencia de residuos infecciosos, tóxicos, químicos y objetos corto-punzantes y principalmente, provoca gran inquietud y percepción de riesgo en la población general. ${ }^{2}$

El riesgo potencial presentado por los residuos sólidos hospitalarios, constituye un problema en términos de salud pública, saneamiento ambiental, enfermedades nosocomiales y epidemiológicos, etc. Es responsabilidad de las instituciones prestadoras de servicios de salud prevenir y contribuir a minimizar este riesgo ambiental. ${ }^{3}$

El manejo de los residuos sólidos en los servicios médicos es un sistema de seguridad sanitaria que se inicia en el punto de generación, para continuar su manejo en las diferentes unidades del hospital, hasta asegurar que llegue a su destino final fuera del establecimiento, para su tratamiento o disposición adecuada, y, responde a un mandato imperativo de la necesidad diaria de minimizar y controlar los riesgos que se derivan del manejo de estos residuos para proteger a la población hospitalaria, es decir, no se restringe sólo a controlar los riesgos sino a lograr minimizar éstos riesgos desde el punto de origen.

El nexo entre la salud humana y el ambiente ha sido reconocido desde hace mucho tiempo. Sin lugar a dudas, la salud humana depende de la voluntad y la capacidad de una sociedad para mejorar la interacción entre la actividad humana y el ambiente químico, físico y biológico. Esto debe hacerse de manera que promocione la salud humana y prevenga la enfermedad, manteniendo el equilibrio y la integridad de los ecosistemas, y evitando comprometer el bienestar de las futuras generaciones. ${ }^{4}$

Las instituciones de salud constituyen ambientes de trabajo especiales, en los que el trabajador se expone a varias enfermedades infecciosas, tanto en la asistencia directa al paciente como en otras áreas, específicamente en aquellas en que se manejan muestras, desechos y algunos fómites, pero no solo este tipo de trabajador, sino otros que laboran en áreas administrativas de estos centros y se exponen indirectamente, así como los visitantes, la comunidad circundante y el medio ambiente. ${ }^{5}$

De igual manera, la Organización Panamericana de la Salud indica que:

Los desechos peligrosos generados en los establecimientos de salud representan un grave problema que incide en la alta tasa de enfermedades infecciosas que registran los países de América Latina. Su potencial patogénico y la ineficiencia de su manejo, incluida la generación, manipulación, inadecuada segregación y la falta de tecnología para su tratamiento y disposición final, constituyen un riesgo para la salud de la comunidad hospitalaria y la población en general". La OPS nos propone poner en regimiento su esquemática que se encuentra en la "Guía para el manejo interno de residuos sólidos en centros de atención de salud (OPS, 2 1994) con la finalidad de controlar y reducir los riesgos para la salud debido a la exposición a los desechos peligrosos. $^{6}$

El manejo de los desechos de instituciones de salud en Cuba es uno de los aspectos de la gestión hospitalaria que ha ido tomando importancia e interés en los últimos años. Es considerado por algunos autores como una herramienta de gestión que garantiza una seguridad sanitaria y ambiental, la cual se inicia desde la fuente de generación, para continuar su manejo en las diferentes áreas de la institución, hasta asegurar que llegue a su destino final fuera del establecimiento, para su tratamiento o disposición adecuada. $^{7}$

En Cuba, existe un marco legal que reglamenta la gestión de los desechos de instituciones de salud. La Norma Cubana NC 530:2009 establece los requisitos sanitarios y ambientales en el manejo de los desechos sólidos en instituciones de salud. Plantea que "se precisa del establecimiento de un sistema seguro de manejo de los desechos sólidos en las instituciones de salud destinado a garantizar la adecuada higiene y seguridad para los trabajadores de la salud, los pacientes y la comunidad general, que conduzcan a la implementación de políticas de reducción de la generación de desechos y al incremento del reciclaje". ${ }^{8}$

Los problemas asociados a los desechos principalmente sólidos generados por las instituciones de salud, han sido motivo de preocupación de diferentes organizaciones internacionales y del Ministerio de Salud Pública de Cuba. Como parte del esfuerzo que se realiza por lograr la seguridad y la salud de los trabajadores de este sector, el cuidado del ambiente y la excelencia en los servicios de salud, el Instituto Nacional de Higiene, Epidemiología y Microbiología (INHEM), en colaboración con la Organización Panamericana de la Salud (OPS), teniendo como sede a instituciones de los tres niveles de atención a la salud, llevó a efecto una investigación en el período 2008-2011 que permitió establecer y perfeccionar los planes institucionales de manejo de dichos desechos en las instituciones participantes, con vistas a optimizar su gestión ambiental y garantizar así la mejora de los servicios de salud que brindan. ${ }^{9}$ Por otra parte, se estableció la Resolución 136/2009 del Ministerio de Ciencia, Tecnología y Medio Ambiente (CITMA) establece el Reglamento para el manejo integral de desechos peligrosos. ${ }^{10}$

El principal problema relacionado con el manejo de los desechos biológicos peligrosos procedentes de instalaciones de salud pública radica en la ausencia de alternativas de tratamiento de estos desechos, que sean ambientalmente compatibles y económicamente viables y que respondan al supuesto de que el generador del desecho es el encargado de su manejo interno en la instalación, su tratamiento y sus disposiciones finales en condiciones de seguridad. ${ }^{11}$ 
Según la UNESCO, la Educación Ambiental "es la reorientación y articulación de diversas disciplinas y experiencias educativas que facilitan la percepción integrada del medio ambiente, haciendo posible una acción más racional y capaz de responder a las necesidades sociales". Su principal objetivo es transmitir conocimientos, formar valores, desarrollar competencias y comportamientos que pueden favorecer la comprensión y la solución de los problemas ambientales $^{1}$ de lo anteriormente se desprende la necesidad primordial de realizar una investigación con el objetivo de evaluar el impacto de una educación ambiental en el manejo de los residuales sólidos peligrosos en el Hospital Universitario "Mártires del 9 de Abril".

\section{Métodos}

Se realizó una investigación cuasiexperimental de intervención con antes y después en el Hospital Universitario "Mártires del 9 de Abril" del municipio de Sagua la Grande durante el año 2019. La población de estudio estuvo constituida por los 7 servicios de mayor generación de residuales y 269 trabajadores que intervienen en el manejo de los residuales sólidos peligrosos en el ambiente hospitalario que cumplieron los criterios de inclusión y la totalidad de trabajadores del departamento de Epidemiología (Epidemiólogo, Técnico de Higiene y Epidemiología, 2 Enfermeras de Vigilancia Epidemiológica), de esta institución durante el año 2019. La muestra se seleccionó a partir de un muestreo no probabilístico por criterios.

Tabla 1: Distribución porcentual de las características generales de los trabajadores que intervienen en el manejo de los residuales sólidos peligrosos. Hospital Universitario "Mártires del 9 de Abril” de Sagua la Grande. 2019

\begin{tabular}{|l|l|c|c|}
\hline \multicolumn{2}{|c|}{ Características } & Nro & $\%$ \\
\hline \multirow{3}{*}{ Ocupación } \\
\hline Grupo 1 & Personal administrativo & 10 & 3.17 \\
\hline Grupo 2 & Médicos & 60 & 22.30 \\
\cline { 2 - 4 } & Enfermeros & 143 & 53.15 \\
\cline { 2 - 4 } & Técnicos de laboratorio & 26 & 9.66 \\
\hline Grupo 3 & Auxiliares generales Servicios de riesgos & 30 & 11.15 \\
\hline \multirow{3}{*}{} & Masculino & 95 & 35.31 \\
\hline & Femenino & 42 & 174.68 \\
\hline & Nefrología y hemodiálisis & 68 & 25.27 \\
\hline & Salón de operaciones & 64 & 23.79 \\
\hline & Salón de partos y cesárea & 35 & 13.01 \\
\hline & Cuerpo de guardia & 3 & 1.11 \\
\hline & Consulta de Quimioterapia & 29 & 10.78 \\
\hline & Laboratorios & 26 & 9.66 \\
\hline & Atención al grave &
\end{tabular}

Fuente: Encuestas

\section{Criterios de inclusión:}

- Personal que labora en los servicios en el momento de la investigación.
- Voluntariedad para participar en el estudio.

- Trabajadores que intervienen directamente en el manejo de los residuales sólidos peligrosos

\section{Criterios de exclusión:}

- Personal que no labora en el momento de la investigación.

- No voluntariedad para participar en el estudio.

- Trabajadores que no intervienen directamente en el manejo de los residuales sólidos peligrosos

\subsection{Instrumentos y técnicas utilizadas}

Obtención de la información.

Se realizó una revisión documental sobre el tema a partir de bibliografía actualizada. En relación con la estructura, la generación, envasado, almacenamiento, transporte, tratamiento y disposición final de los residuales sólidos peligrosos así como los documentos y registros del departamento de Higiene y Epidemiología y se consultó además la plantilla de recursos humanos (Tabla 2).

Tabla 2: Distribución porcentual de la evaluación de las Dimensiones Estructura, Proceso y Resultado del manejo de los residuales sólidos peligrosos. Hospital Universitario "Mártires del 9 de Abril” de Sagua la Grande. 2019

\begin{tabular}{|c|c|c|}
\hline \multirow{2}{*}{\multicolumn{3}{|c|}{$\begin{array}{c}\text { Criterios evaluados en la dimensión Estructura } \\
\end{array}$}} \\
\hline & & \\
\hline $\begin{array}{l}\text { 1-Cobertura de personal que trabaja en el manejo de los } \\
\text { residuales sólidos peligrosos. }\end{array}$ & $77.10 \%$ & Insatisfactorio \\
\hline $\begin{array}{l}\text { 2-Disponibilidad de barreras físicas de protección para el } \\
\text { personal }\end{array}$ & $67.44 \%$ & Insatisfactoric \\
\hline $\begin{array}{l}\text { 3-Disponibilidad de materiales e insumos necesarios para el } \\
\text { acondicionamiento, la segregación y almacenamiento primario }\end{array}$ & $57.70 \%$ & Insatisfactori \\
\hline $\begin{array}{l}\text { 4. Condiciones estructurales del local de almacenamiento } \\
\text { intermedio }\end{array}$ & $48.73 \%$ & Insatisfactorio \\
\hline 5. Condiciones estructurales del Transporte Interno & $86.10 \%$ & Insatisfactoric \\
\hline 6. Condiciones estructurales del local de almacenamiento final & $76.45 \%$ & \\
\hline 7. Disponibilidad de método de tratamiento & $0 \%$ & Insatisfactorio \\
\hline 8. Disponibilidad de recolección externa & $100 \%$ & Satisfactorio \\
\hline 9. Disponibilidad de disposición final & $100 \%$ & Satisfactorio \\
\hline $\begin{array}{l}\text { 10. Inmunización del personal según el nivel de riesgo } \\
\text { biológico. (res } 103 / 2002 \text { ) }\end{array}$ & $100 \%$ & Satisfactorio \\
\hline 11. Cumplimiento del chequeo pre-empleo y periódico. & $100 \%$ & Satisfactorio \\
\hline 12. Existencia del Plan de $n$ & $100 \%$ & Satisfactorio \\
\hline 13. Existencia de la licencia ambiental & $100 \%$ & Satisfactorio \\
\hline \multicolumn{3}{|l|}{$\begin{array}{c}\text { Criterios evaluados en la dimensión Proceso } \\
\end{array}$} \\
\hline 1. Cumplimiento adecuado de la utilización de barreras físicas. & $53.19 \%$ & Insatisfactorio \\
\hline $\begin{array}{l}\text { 2. Cumplimiento del adecuado uso de materiales e insumos } \\
\text { necesarios para la segregación y almacenamiento primario }\end{array}$ & $56.87 \%$ & Insatisfactorio \\
\hline 3. Uso adecuado de locales para el almacenamiento intermedio & $45.72 \%$ & Insatisfactorio \\
\hline 4- Cumplimiento del adecuado uso de Transporte Interno & $67.23 \%$ & Insatisfactorio \\
\hline 5. Uso adecuado de locales para el almacenamiento final & $40.03 \%$ & Insatisfactorio \\
\hline $\begin{array}{l}\text { 6. Uso adecuado de método de tratamiento de los residuales } \\
\text { sólidos peligrosos. }\end{array}$ & $36.71 \%$ & Insatisfactorio \\
\hline $\begin{array}{l}\text { 7. Cumplimiento del adecuado uso de equipo(s) según método } \\
\text { de tratamiento }\end{array}$ & $55.62 \%$ & Insatisfactori \\
\hline 8. Cumplimiento adecuado recolección externa & $87.34 \%$ & Insatisfactori \\
\hline 9. Cumplimiento adecuado de disposición final & $87.30 \%$ & Insatisfactori \\
\hline \multicolumn{3}{|l|}{$\begin{array}{c}\text { Criterios evaluados en la dimensión Resultado } \\
\end{array}$} \\
\hline $\begin{array}{l}\text { 1-Proporción de trabajadores enfermos por contacto con los } \\
\text { residuales sólidos peligrosos. }\end{array}$ & $0 \%$ & Satisfactorio \\
\hline $\begin{array}{l}\text { 2-Proporción de accidentes en los trabajadores por contacto con } \\
\text { los residuales sólidos peligrosos. }\end{array}$ & $0 \%$ & Satisfactorio \\
\hline $\begin{array}{l}\text { 3-Incidentes en relación con el manejo de los residuales } \\
\text { sólidos peligrosos. }\end{array}$ & $4.55 \%$ & Insatisfactori \\
\hline $\begin{array}{l}\text { 4-Satisfacción del personal que labora en el manejo de los } \\
\text { residuales sólidos peligrosos. }\end{array}$ & $55.39 \%$ & factori \\
\hline
\end{tabular}

Fuente: Guía de Revisión Documental y encuestas

Se realizaron guías de observación para evaluar algunos indicadores de la estructura y de proceso (Tabla 3 y 4). Se realizó entrevista no estructurada al epidemiólogo del 
hospital y se aplicó un cuestionario al equipo de epidemiología de la institución (Anexo 5) y se plantearon diferentes criterios, con indicadores y estándares para evaluarlos. Para medir el nivel de conocimiento o capacitación de personal que trabaja en el manejo de los residuos sólidos se aplicó un cuestionario a este personal (Anexo 6) y se evaluaron las respuestas de cada Ítem según clave de calificación para medir nivel de conocimientos sobre manejo de residuales sólidos en el ambiente hospitalario (Anexo 7).

Tabla 3: Distribución porcentual de la evaluación general antes y después de aplicado el programa del Manejo de los residuales sólidos peligrosos en el Hospital Universitario "Mártires del 9 de Abril" de Sagua la Grande. 2019

\begin{tabular}{|c|c|c|c|c|}
\hline \multirow{2}{*}{ Dimensiones } & \multicolumn{2}{|c|}{ Antes } & \multicolumn{2}{c|}{ Después } \\
\cline { 2 - 5 } & $\%$ Satisfactorio & $\begin{array}{c}\text { Evaluación } \\
\text { General }\end{array}$ & $\%$ Satisfactorio & $\begin{array}{c}\text { Evaluación } \\
\text { General }\end{array}$ \\
\hline $\begin{array}{c}\text { Estructura } \\
13 \text { indicadores }\end{array}$ & $46.15 \%$ & Insatisfactoria & $76.92 \%$ & Satisfactoria \\
\hline $\begin{array}{c}\text { Proceso } \\
9 \text { indicadores }\end{array}$ & $0 \%$ & Insatisfactoria & $88.88 \%$ & Satisfactoria \\
\hline $\begin{array}{c}\text { Resultados con } 4 \\
\text { indicadores }\end{array}$ & $50.00 \%$ & Insatisfactoria & $75.00 \%$ & Satisfactoria \\
\hline Evaluación Final & 30.76 & Insatisfactoria & $80.76 \%$ & Satisfactoria \\
\hline
\end{tabular}

Fuente: Guía de Revisión Documental y encuestas

Tabla 4: Distribución porcentual según nivel de conocimientos antes y después de aplicado el programa del Manejo de los residuales sólidos peligrosos en el Hospital Universitario "Mártires del 9 de Abril" de Sagua la Grande. 2019

\begin{tabular}{|l|c|c|c|c|}
\hline \multirow{2}{*}{\multicolumn{1}{|c|}{ Parámetros evaluados }} & \multicolumn{4}{c|}{ Conocen } \\
\cline { 2 - 5 } & \multicolumn{2}{|c|}{ Antes } & \multicolumn{2}{c|}{ Después } \\
\cline { 2 - 5 } & Nro & $\begin{array}{c}\% \\
(\mathrm{~N}=269)\end{array}$ & Nro & $\begin{array}{c}\% \\
(\mathrm{~N}=269)\end{array}$ \\
\hline Etapas del manejo de los residuales peligrosos & 150 & 55.76 & 245 & 91.07 \\
\hline $\begin{array}{l}\text { Principales problemas ambientales en cada una de } \\
\text { las etapas del manejo de los residuales peligrosos }\end{array}$ & 102 & 37.91 & 263 & 97.76 \\
\hline $\begin{array}{l}\text { Riesgos para la salud de la población hospitalaria } \\
\text { en cada uno de las etapas del manejo de los } \\
\text { residuales peligrosos }\end{array}$ & 134 & 49.81 & 243 & 90.33 \\
\hline $\begin{array}{l}\text { Marco legal existente con relación al manejo de los } \\
\text { residuales peligrosos }\end{array}$ & 68 & 25.27 & 260 & 96.65 \\
\hline
\end{tabular}

Fuente: Encuestas

Sobre el resultado de la implementación de las normas y medidas establecidas para el manejo de los residuales solidos peligrosos, se hizo una revisión de los documentos relacionados con las enfermedades, accidentes e incidentes relacionados con los trabajadores por contacto con los residuales solidos peligrosos (Tabla 2), se entrevistó al epidemiólogo del hospital y se aplicó un cuestionario al equipo de epidemiología de la institución (Tabla 5) y una encuesta de satisfacción sobre la percepción de calidad de la atención del personal que labora en el manejo de los residuos sólidos peligrosos e igualmente se plantearon diferentes criterios, con indicadores y estándares para evaluarlos.
Tabla 5: Distribución porcentual según impacto del programa de educación ambiental en el manejo de los residuales sólidos peligrosos en el Hospital Universitario "Mártires del 9 de Abril" de Sagua la Grande. 2019

\begin{tabular}{|l|c|c|}
\hline Impacto del programa de educación ambiental & No & \% \\
\hline Bueno & 256 & 95.16 \\
\hline Regular & 13 & 4,83 \\
\hline Malo & 0 & 0,00 \\
\hline Total & $\mathbf{2 6 9}$ & $\mathbf{1 0 0 , 0 0}$ \\
\hline
\end{tabular}

Fuente: Programa de educación ambiental

Los criterios, indicadores y estándares están evaluados en un diseño evaluativo aplicado anteriormente, los instrumentos a utilizar fueron validados anteriormente y otros se validaron para esta investigación por expertos (másteres y epidemiólogos) con experiencia en el tema de evaluación y en el manejo de los residuales sólidos peligrosos en el ambiente hospitalario.

\section{Operacionalización de los criterios:}

Evaluación general de la Dimensión Estructura, proceso y resultado:

Satisfactoria: Si el $95 \%$ o más de los indicadores recibieron evaluación Satisfactoria

Insatisfactoria: Si menos del $95 \%$ de los indicadores recibieron evaluación Satisfactoria

En la segunda etapa se procesó y se analizó la información obtenida a partir de los datos en el análisis de los documentos, la observación y el cuestionario, y se diseñó la propuesta de un programa de educación ambiental en el manejo de los residuales sólidos peligrosos.

Estructura del Programa de educación ambiental

Nivel de aplicación: Servicios de mayor generación de residuales y trabajadores que intervienen en el manejo de los residuales sólidos peligrosos en el ambiente hospitalario

Puesto en marcha por: Profesionales de enfermería vigilantes epidemiológicas

Objetivo General: implementar un programa de educación ambiental para mejorar el nivel de conocimientos en el manejo de residuos sólidos en personal de salud así como identificar y resolver los problemas ambientales en cada una de las etapas del manejo de los residuales peligrosos

Temas del programa de educación ambiental

1) Etapas del manejo de los residuales peligrosos

2) problemas ambientales en cada una de las etapas del manejo de los residuales peligrosos

3) Riesgos para la salud de la población hospitalaria en cada uno de las etapas del manejo de los residuales peligrosos

4) Marco legal existente con relación al manejo de los residuales peligrosos

En la 3ra etapa, después de transcurridos 6 meses de haber impartido la capacitación los investigadores aplicaron nuevamente la encuesta inicial para evaluar el nivel de conocimientos que sobre residuales solidos hospitalarios 
tenían los estudiados y establecer comparaciones con los resultados iniciales.

Procesamiento de la información:

A partir de los distintos instrumentos empleados se obtuvo la base de datos donde se procesó la información estadísticamente mediante el programa SPSS lo cual hizo factible la elaboración de un resumen y el análisis de la información que quedó plasmado en tablas. Se utilizaron los métodos estadístico descriptivo y la distribución de frecuencias. Los textos se trabajaron con Microsoft Excel y las tablas se realizarán con Microsoft Word.

Aspectos éticos:

Para este estudio se solicitó el consentimiento informado del personal de salud (Anexo 1), incluido en el estudio, de ser tomados como miembros de la investigación, se les explicó correctamente qué, para qué y por qué se hace el estudio y que sería libre de elegir su participación en la investigación.

\section{Resultados}

Para la evaluación del manejo de los residuales sólidos peligrosos en el Hospital Universitario "Mártires del 9 de Abril" de Sagua la Grande se comenzó analizando las características generales del personal, disponibles en la tabla 1,siendo el $64.68 \%$ del sexo femenino, de ocupación enfermeros el $53.15 \%$, médicos $22.30 \%$ y auxiliares generales el $11.15 \%$. el $25.27 \%$ trabajadores del salón de operaciones.

Para la evaluación de los indicadores relacionados con la estructura, proceso y resultado disponibles en la tabla 2 , obsérvese que en los 13 criterios evaluados en la dimensión estructura el, 6 cumplieron con el estándar establecido que representan el $46.15 \%$ de los criterios con evaluación satisfactoria, por lo cual la evaluación integral de la dimensión estructura resultó insatisfactoria. Para la evaluación del componente proceso indicadores relacionados con el proceso de segregación, envasado, almacenamiento, transporte, tratamiento y disposición final de los residuales sólidos; la evaluación integral de la dimensión proceso resultó no adecuada. Los 9 criterios no cumplieron con el estándar establecido representando el $100 \%$ con evaluación insatisfactoria, quedando de forma general por debajo del $95 \%$.

En la tabla 2 también se muestran los indicadores de la dimensión resultados, de los 4 indicadores analizados, cumplieron con el estándar establecido 2 de ellos, representando el $50.00 \%$, y fue evaluada esta dimensión de insatisfactoria.

La tabla 3 se expone la evaluación final del manejo de los residuales peligrosos en el Hospital Universitario "Mártires del 9 de Abril" de Sagua la Grande, antes y después del programa de educación ambiental. Antes de aplicado el programa de 26 criterios evaluados, solo 8 recibieron evaluación satisfactoria para un $30.76 \%$, después de aplicado el programa recibieron evaluación satisfactoria 21 criterios para un $80.76 \%$.
Las tres dimensiones estructura con un $76.92 \%$, proceso con un $88.88 \%$ y resultado con un $75.00 \%$ fueron evaluadas como satisfactorias después de aplicado el programa, por lo cual resultó la evaluación general de esta institución satisfactoria.

En cuanto a las respuestas correctas sobre temas relacionados con los residuales solidos peligrosos se observa en la tabla 4 que antes de aplicado el programa de educación ambiental excepto en las etapas del manejo de los residuales solidos peligrosos el resto de los aspectos evaluados no alcanzó más del $49.81 \%$, después de impartido el programa alcanzó más del $90.33 \%$ en todos los aspectos evaluados.

La tabla 5 muestra el impacto del programa de educación ambiental en el manejo de los residuales solidos peligrosos que resulto ser positivo, se logró elevar el nivel de conocimiento de los trabajadores en el manejo de los residuales así como mejorar los criterio evaluados en las 3 dimensiones, estructura, proceso y resultado.

\section{Discusión}

En las normas de bioseguridad para el manejo de desechos biológicos peligrosos, además de las precauciones universales de bioseguridad, deben cumplirse requisitos que incluyen utilizar, de forma permanente, los elementos de protección personal; evacuar los desechos y anudar las bolsas que los contienen; no introducir las manos dentro del recipiente pues podría ocasionar accidentes del trabajo por cortaduras, pinchazos o contacto con material contaminado; evitar vaciar desechos de un recipiente a otro; considerar todo material que esté envasado como contaminado; asegurarse de que todos los desechos cortopunzantes y de riesgo biológico hayan sido sometidos a previo proceso de desinfección y mantener en óptimas condiciones de higiene los recipientes, carros de transporte, áreas de almacenamiento y áreas de disposición final de desechos. ${ }^{12}$

Es responsabilidad de la administración crear todas las condiciones y garantizar los materiales e insumos para el acondicionamiento de los residuos sólidos, etapa violada y generalmente olvidada por los investigadores de artículos científicos. $^{10}$

El acondicionamiento a la preparación de los servicios y áreas hospitalarias con los materiales e insumos necesarios para segregar los residuos de acuerdo a los criterios técnicos establecidos según el volumen de producción y clase de residuos que genera cada uno de los servicios de salud y los materiales necesarios para su descarte. ${ }^{13}$

En estudio realizado por Pardo M y refiriéndose a las instituciones de salud plantea que la gestión interna consiste en la planeación e implementación articulada de todas y cada una de las actividades realizadas al interior de la entidad generadora de residuos hospitalarios y similares, con base en este manual; incluyendo las actividades de generación, segregación en la fuente, desactivación, movimiento interno, almacenamiento y entrega de los residuos al prestador del servicio especial de aseo, sustentándose en criterios técnicos, económicos, sanitarios y ambientales; 
asignando recursos, responsabilidades y garantizando, mediante un programa de vigilancia y control el cumplimiento del Plan. ${ }^{14}$

Junco Díaz y Rodríguez Sordia plantean que para el envasado de los desechos generados de acuerdo con sus características físicas y biológico-infecciosas, se debe contar con recipientes apropiados para cada tipo de desecho. El tamaño, peso, color, forma y material deben garantizar una apropiada identificación, facilitar las operaciones de transporte y limpieza, ser herméticos para evitar exposiciones innecesarias, y estar integrados a las condiciones físicas y arquitectónicas del lugar. Los recipientes, las bolsas y los lugares donde estos se ubican deben tener un código de colores e impresos visibles que indiquen el tipo de desechos que representan (rojo para los infeccioso, negro o blanco para los comunes y verde o amarillo para los especiales). ${ }^{15}$

El método de tratamiento a aplicar será el que menor daño represente a la población hospitalaria y al medio ambiente, los recomendados son: enterramiento controlado, esterilización por autoclave, incineración y desinfección por microondas. ${ }^{16}$

Otros autores plantean en su investigación que el tratamiento de los desechos sólidos peligrosos se realizaba por incineración, con un equipo antiguo que se encontraba en mal estado deficiente desde el punto de vista técnico, coincidiendo con los resultados obtenidos en esta investigación. ${ }^{17,18}$

Es factible el desarrollo de actividades de investigación para evaluar los conocimientos de los profesionales y los técnicos en relación a contaminantes biológicos que permitan a la vez profundizar más en el conocimiento sobre la bioseguridad en salud. ${ }^{19}$

Según Junco en su investigación, en la atención secundaria y terciaria el tratamiento utilizado era la incineración mediante incineradores de una sola cámara con un nivel de combustión bajo en la mayoría de los casos mediante un servicio brindado por otro centro o en la propia institución, con un equipo antiguo y deficiente desde el punto de vista técnico, por lo que el resultado era una cremación incompleta. $^{20}$

Los hallazgos insatisfactorios de las prácticas sugieren la necesidad de mejorar los programas educativos, las estrategias de vigilancia, inspección y control, la sensibilización frente a los riesgos sanitarios, ambientales, legales y económicos derivados de la mala praxis y en general la optimización de todos los procesos inherentes al manejo integral de los residuos hospitalarios. ${ }^{21}$

\section{Conclusiones}

El programa de educación ambiental elevó el nivel de conocimiento de los trabajadores en el manejo de los residuales peligrosos y contribuyó a mejorar los criterios evaluados en las dimensiones, estructura, proceso y resultado en el ambiente hospitalario

\section{Fuente de Financiamiento}

Este estudio es autofinanciado.

\section{Conflicto de Intereses}

No existen conflictos personales, profesionales, financieroso de otro tipo.

\section{Consentimiento Informado}

Los autores cuentan con el consentimiento informado de los pacientes para la investigación, la publicación del caso y sus imágenes.

\section{Referencias Bibliográficas}

1. Ventura Esquivel ER. Impacto de una educación ambiental para mejorar conocimientos en manejo de residuos sólidos en personal de salud. Microred Ate-II; 2019.

2. Salas RG, Arroyo RCM, Río MV. Eficacia del manejo de los residuos en el Hospital San Vicente de Paul, ciudad de Ibarra. Revista UNIANDES Episteme. 2017;4(1):1-9.

3. Carranza JD. Evaluación y recomendaciones del manejo de residuos hospitalarios en un centro de asistencia de salud del área norcentro de la ciudad de Guatemala;. Available from: http://www.bvsde.paho.org/bvsaidis/mexico26/ iv-007.pdf.

4. Suárez Pita MdlA, Junco Díaz R. Plan institucional de manejo de los desechos sólidos, una herramienta para la gestión hospitalaria. Rev Cubana Hig Epidemiol. 2012;50(3). Available from: http://scielo.sld.cu/scielo.php?script=sci_ arttext\&pid=S1561-30032012000300015\&lng=es.

5. Vera Núñez D. Efectividad de Guía de Buenas Prácticas en la bioseguridad hospitalaria. Rev Cubana Enferm. 2017;33(1). Available from: http://www.revenfermeria. sld.cu/index.php/enf/article/view/1208.

6. Carrillo Saracay AA. Evaluación de las rutas de evacuación de los desechos peligrosos hospitalarios generados en sus diferentes áreas. Hospital de la Policía Nacional No 2 de Guayaquil. [Tesis]; 2018. Available from: http: //repositorio.ug.edu.ec/handle/redug/29529.

7. Trincado Agudo MT, Ramos Valle I, Vázquez Adán Y, Guillén Fonseca M. Evaluación de las normas de bioseguridad en el servicio de hemodiálisis del Instituto de Nefrología "Dr. Rev Cubana Hig Epidemiol. 2009;49(3). Available from: http://scielo.sld.cu/scielo.php?script=sci_ arttext\&pid=S1561-30032011000300005\&lng=es.

8. Oficina Nacional Normalización. Desechos sólidos: manejo de desechos sólidos de instituciones de salud - requisitos sanitarios y ambientales. La Habana; 2009. NC 530:2009.

9. Rodríguez Bertheau AM, Martínez Varona M, Martínez Rodríguez I, Fundora Hernández H, Guzmán Armenteros T. Desarrollo tecnológico, impacto sobre el medio ambiente y la salud. Rev Cubana Hig Epidemiol. 2011;49(2). Available from: http://scielo.sld.cu/scielo.php?script=sci_ arttext\&pid=S1561-30032011000200016\&lng=es.

10. Ministerio Ciencia Tecnología y Medio Ambiente. Reglamento para el manejo integral de desechos peligrosos. La Habana: CITMA; 2009. Resolución 136/2009. 
11. Gómez T, Suárez Y, González O, Béquer L, Guirado O, Aparicio J. Implementación del sistema de gestión del riesgo biológico en la Universidad Médica de 18. Villa Clara. Rev Cubana Hig Epidemiol. 2012;50(2). Available from: http://scielo.sld.cu/scielo.php?script=sci_ arttext\&pid=S1561-30032010000100007\&lng=es.

12. Verdera Hernández J, Bermúdez Pérez R. Desechos 19. Reyes Roque AC, Benítez Pérez MO, Fraga Rodríguez A. biológicos peligrosos. En: Bioseguridad básica. La Habana: Ciencias Médicas; 2011. Available from: http://www.bvs.sld.cu/libros/bioseguridad_basica/ bioseguridad_basica_completo.pdf.

13. Urquiza Álvarez J. Evaluación del manejo de los residuales sólidos peligrosos en el ambiente hospitalario 20. [tesis]. Universidad de Ciencias Médicas Dr. Serafín Ruiz de Zárate Ruiz. Villa Clara; 2014.

14. Lizeth Pardo M. Propuesta de una metodología para la ubicación de depósitos intermedios y/o finales de residuos no deseados generados por centros de atención hospitalarios. Universidad del Valle; 2011. Available from: http://bibliotecadigital. univalle.edu.co/xmlui/bitstream/handle/10893/8102/CB0449625.pdf?sequence=1.pdf.

15. Junco Díaz RA, Rodríguez Sordia DS. Desechos Hospitalarios: Aspectos Metodológicos de su Manejo. Instituto Nacional de Higiene, Epidemiologia y Microbiología. Rev Cubana Hig Epidemiol. 2015;38(2):122-6.

16. Gayoum Saad SA. Management of hospitals solid waste in khartoum state. Environ Monit Assess. 2013:185. Available from: http://web.a.ebscohost.com/ehost/ pdfviewer/pdfviewer?sid=26915eb6-935d-4346-9734db3b21782e0d\%40sessionmgr4004\&vid=0\&hid $=4204$.

17. Junco Díaz RA, Prieto Díaz V, Weng Alemán Z, Suárez Pita MT. Manejo de los desechos sólidos en el sector de la salud: necesidad de un plan institucional. In: Memorias
Convención Internacional de Salud Pública. Cuba Salud 2013;. La Habana 3-7 de diciembre de 2013.

. Junco Díaz RA, Prieto Díaz V, Weng Alemán Z, Suárez Pita MT. Caracterización de los desechos sólidos en instituciones de salud como etapa básica para el plan de manejo; 2015.

La disposición de los desechos peligrosos: un desafío para la seguridad biológica. CARTA AL DIRECTOR. Hospital Militar "Comandante Manuel Fajardo Rivero". vol. 10. Santa Clara, Villa Clara, Cuba: Acta Médica del Centro; 2016.

. Junco Díaz RA, Prieto Díaz V, Weng Alemán Z, Suárez Pita MT; 2015. .

21. Quinto M, Jaramillo Pérez LM, Cardona Arias J. Conocimientos y prácticas de los trabajadores de un hospital sobre el manejo de residuos hospitalarios, Chocó, Colombia, 2012. vol. 26. MÉD UIS; 2013. Available from: http://www. scielo.org.co/scielo.php?script=sci_pdf\&pid=S012103192013000100002\&lng=en\&nrm=iso\&tlng=es.

Recibido: 12 de febrero de 2020

Aceptado: 25 de junio de 2020 
